\title{
Down-regulation of caveolin-1 in mouse Lewis lung cancer P29 is a causal factor for the malignant properties in a high-metastatic subline
}

\author{
QING ZHANG ${ }^{1}$, KEIKO FURUKAWA ${ }^{1}$, HO-HSIANG CHEN ${ }^{1,2}$, REIKO FUJINAWA ${ }^{3}$, \\ YASUNORI KOZUTSUMI ${ }^{3}$, AKEMI SUZUKI ${ }^{3}$, TAKESHI URANO ${ }^{1}$ and KOICHI FURUKAWA ${ }^{1}$
}

Departments of ${ }^{1}$ Biochemistry II, ${ }^{2}$ Surgery II, Nagoya University Graduate School of Medicine, 65 Tsurumai, Showa-ku,
Nagoya 466-0065; ${ }^{3}$ Supra-Biomolecular Sytstem Research Group, Riken Frontier Research System, Wako 351-0198, Japan

Received January 24, 2006; Accepted March 16, 2006

\begin{abstract}
In order to analyze molecular mechanisms for cancer metastasis, we established a high-metastatic subline H7-Lu from a subline $\mathrm{H} 7$ of mouse Lewis lung cancer (P29) by repeated injection into tail veins. H7-Lu exhibited increased proliferation and invasion activity. Analysis of gene expression profiles between the parent $\mathrm{H} 7$ and $\mathrm{H} 7-\mathrm{Lu}$ revealed that several genes were down-regulated in H7-Lu. One of them, caveolin-1, was a component of lipid/rafts. After confirming the down-regulation of caveolin-1 mRNA by real-time RT-PCR and reduction of the protein by immunoblotting, respectively, H7 was transfected with siRNA for caveolin-1 to examine the role of caveolin-1 in H7-Lu. mRNA of the caveolin-1 gene was suppressed to approximately one third of the original level in $\mathrm{H} 7$ cells transfected with siRNA. The transfectant cells showed significantly increased cell proliferation and motility when analyzed by MTT assay and scratching wound healing assay, respectively. In the siRNA-transfectant cells, both ERK1/2 and Akt showed stronger phosphorylation than the mock-transfectant cells indicating that both of these signaling pathways were activated in caveolin-1-suppressed cells. These situations seem to reflect some aspects of the cellular changes in the high metastatic subline H7-Lu. Thus, down-regulation of caveolin-1 in a high-metastatic subline of Lewis lung cancer as defined by DNA array is really a causal factor for the increased malignant properties.
\end{abstract}

\section{Introduction}

Although the development of metastasis is a major cause of death in many cancers, mechanisms for the acquisition of metastatic potential are not well understood. Recently, a

Correspondence to: Dr Koichi Furukawa, Department of Biochemistry II, Nagoya University Graduate School of Medicine, Tsurumai, Showa-ku, Nagoya 466-0065, Japan

E-mail: koichi@med.nagoya-u.ac.jp

Key words: caveolin, raft, metastasis, microdomain, DNA array number of molecules involved in metastasis have been identified and their functions have been well studied (1). Cell adhesion (2), angiogenesis (3) and lymph-angiogenesis (4), and protease-mediated migration and invasion (5) have been eagerly investigated.

Microdomains in the cell surface membrane are recognized as an important site for cholesterol metabolism, endocytosis, infection of microorganisms, apoptosis, and signal transduction (6). These microdomains are called caveolae (7), lipid rafts (8), or glycolipid-enriched microdomain (GEM) (9), and we refer to them as GEM/rafts. Little has been known on the roles of GEM/ rafts and caveolin-1, a main component of the microdomain in cancer metastasis, although their implications in cell transformation and oncogenesis have been extensively studied (10).

We established a high-metastatic subline from $\mathrm{H} 7$ derived from mouse Lewis lung cancer P29 (11) by repeatedly injecting into tail veins, and analyzed cellular changes between the parent cells and the high-metastatic subline with gene expression profiling using DNA array. We found that caveolin-1 was strongly down-regulated in the high-metastatic subline H7-Lu. Then, we demonstrated that caveolin-1 is a crucial factor in regulating the malignant properties of cancer cells. This study should be the first to report the role of caveolin- 1 in the regulation of tumor features based on gene expression profiling of metastatic tumor cells.

\section{Materials and methods}

Cell lines and culture. P29, a low-metastatic subline (with s.c. inoculation) of Lewis lung cancer was subcloned to establish monoclonal sublines $\mathrm{H} 7$. H7 was repeatedly injected into C57BL/6 mice intravenously (i.v.), and a high-metastatic subline H7-Lu was established. Cells were maintained in Dulbecco's modified Eagle's medium (DMEM) supplemented with $7.5 \%$ fetal bovine serum (FBS) at $37^{\circ} \mathrm{C}$ in a humidified atmosphere of $95 \%$ air and $5 \% \mathrm{CO}_{2}$.

Antibodies. Anti-ERK1/2 and anti-phosphorylated ERK1/2 antibody, anti-Akt and anti-phosphorylated Akt antibody, and HRP-conjugated anti-rabbit IgG were purchased from Cell Signaling Technology (Beverly, MA). Anti-caveolin-1 
antibody was purchased from Santa Cruz Biotechnology (CA). Anti-ß-actin antibody was purchased from Sigma (St. Louis, MO).

Assay of metastasis. Cells were detached from culture dishes by 5-min treatment with $0.02 \%$ EDTA in PBS, centrifuged and resuspended in $200 \mu \mathrm{l}$ PBS. For the experimental metastasis assay, cells $\left(0.5 \times 10^{6} /\right.$ mouse $)$ were injected into the tail veins of C57BL/6 mice. Mice were sacrificed at 3 weeks after injection, and the number of metastatic nodules on the surface of the lungs was counted. When mice died before this term, the metastatic nodules were counted at death. The metastatic nodules from lungs were isolated and cells were cultured in vitro to be used for the next injection and/or other functional assays.

Gene expression profiling. Expression profiling of genes in the parent cell line $\mathrm{H} 7$ and the high-metastatic subline H7-Lu was performed as described previously (12). Briefly, total RNA was isolated from $\mathrm{H} 7$ and H7-Lu cells ( $1 \times 10^{7}$ cells) by using the mTRAP mRNA isolation kit ${ }^{\mathrm{TM}}$ (Active Motif, Carlsbad, CA). Cy3-dUTP (H7) or Cy5-dUTP (H7-Lu) was incorporated into cDNA by using the CyScribe First-Strand cDNA-labeling kit ${ }^{\mathrm{TM}}$ (Amersham Pharmacia Biotech). Two cDNA samples, labeled with either $\mathrm{Cy} 3$ or $\mathrm{Cy} 5$ fluorescent dyes, were applied competitively to the microarray, which contained 1,000 cDNAs relevant with complex carbohydrate synthesis and lipid metabolism and another 6,400 cDNAs of mouse genes mapped in the UniGene database. A fluorescence image of the microarray was obtained by using an Affymetrix 428 array scanner ${ }^{\text {TM }}$ (Affymetrix, Santa Clara, CA). Data obtained from the scanner were analyzed further by IMAGENE (BioDiscovery, Marina del Rey, CA). The genes whose expression levels were $>2$-fold higher in $\mathrm{H} 7$ than in $\mathrm{H} 7-\mathrm{Lu}$ were selected as down-regulated genes along the acquisition of metastatic potential.

MTT assay. Cells $\left(2 \times 10^{3}\right)$ were seeded into 96-well plates and cultured in DMEM containing 7.5\% FBS. Twenty microliters of $5 \mathrm{mg} / \mathrm{ml}$ MTT (Sigma) in PBS were added to each well. After incubation for $3.5 \mathrm{~h}$ at $37^{\circ} \mathrm{C}, 150 \mu 1$ of $n$-propyl alcohol containing $0.1 \%$ Nonidet $\mathrm{P}-40$ and $4 \mathrm{mM} \mathrm{HCl}$ was added. The color reaction was quantitated using the automatic plate reader Immuno-Mini NJ-2300 ${ }^{\mathrm{TM}}$ (Nihon Intermed, Tokyo, Japan) at $590 \mathrm{~nm}$ with a reference filter of $620 \mathrm{~nm}$.

Matrigel invasion assay. Cells $\left(0.5 \times 10^{6}\right)$ were resuspended in serum-free DMEM and seeded in the upper chamber of Matrigel-coated transwell filters ( $8 \mu \mathrm{m}$ pore) (BectonDickinson, Franklin Lakes, NJ). The plain medium with or without serum was added to the lower chamber and incubated at $37^{\circ} \mathrm{C}$ for $20 \mathrm{~h}$. Non-invading cells remaining on the upper surface of the filter were removed, and the cells that appeared on the lower surface of the filter were fixed with $75 \%$ ethanol for $30 \mathrm{~min}$, then stained and counted under a microscope.

Scratching wound healing motility assays. Cells $\left(1 \times 10^{6}\right)$ were seeded in 3.5-cm culture dishes and cultured for $12 \mathrm{~h}$. Cells were wounded with a pipett tip, rinsed, and incubated in DMEM with $7.5 \%$ FBS. The areas newly occupied with moved cells in the scratched spaces were measured by taking pictures at the time points of $0,6,24,48$ and $72 \mathrm{~h}$ after the scratching. For statistical analysis, the wound distances were measured at 3 randomly defined wound sites in two dishes (total of 6 sites at each time point).

Cell lysis and immunoblotting. Cells $\left(0.5 \times 10^{6}\right)$ were seeded in 6-cm culture dishes and cultured in DMEM containing $7.5 \%$ FBS. Cells were lysed separately in $0.5 \mathrm{ml}$ of ice-cold lysis buffer [20 mM Tris- $\mathrm{HCl}$ (pH 7.5), $150 \mathrm{mM} \mathrm{NaCl}, 1 \mathrm{mM}$ $\mathrm{Na}_{2}$ EDTA, 1 mM EGTA, 1\% Triton X-100, 2.5 mM sodium pyrophosphate, $1 \mathrm{mM}$ ß-glycerophosphate, $1 \mathrm{mM} \mathrm{Na}_{3} \mathrm{VO}_{4}$, $1 \mu \mathrm{g} / \mathrm{ml}$ leupeptin and $1 \mathrm{mM}$ PMSF], and insoluble material was removed by centrifugation at $4^{\circ} \mathrm{C}$ and $15,000 \mathrm{x}$ g for 10 min. Lysates were separated with SDS-PAGE using 10-15\% gels. The separated proteins were transferred onto a polyvinylidene difluoride (PVDF) membrane (Millipore). Blots were blocked with $0.05 \%$ Tween-20 in PBS (PBST) containing $8 \%$ BSA for $1 \mathrm{~h}$. The membrane was first probed for $1 \mathrm{~h}$ with primary antibodies at the dilution suggested by the suppliers. After washing three times with PBST, the blots were then incubated for $1 \mathrm{~h}$ with goat anti-rabbit IgGs or goat antimouse IgGs conjugated with horseradish peroxidase (Cell Signaling) (1:4000). After the membranes were washed three times with PBST, bound conjugates were visualized with an ECL detection system ${ }^{\mathrm{TM}}$ (Perkin-Elmer Life Science, Boston, MA).

siRNA against caveolin-1 and transfection. The siRNA for caveolin-1 was generated after the screening of three small interfering RNAs (siRNAs). The 25-mer candidate target sequences were selected from the open reading frame of mouse caveolin-1 (GenBank accession no. NM_007616), corresponding to nucleotides 203 to 228,473 to 498 and 481 to 506 (relative to the start codon). These siRNA sequences contained 5'-AAUCUCCUUGGUGUGCGCGUCAUAC-3' and 5'GUAUGACGCGCACACCAAGGAGAUU-3', 5'-AAUCA GGAAGCUCUUGAUGCACGGU-3' and 5'-ACCGUGC AUCAAGAGCUUCCUGAUU-3', 5'-UGAAUCUCAA UCAGGAAGCUCUUGA-3' and 5'-UCAAGAGCUU CCUGAUUGAGAUUCA-3' (Invitrogen), respectively. We used nonspecific control fluorescein-labeled luciferase GL2 Duplex provided by Dharmacon Research (Chicago, IL). For the siRNA transfection, $\mathrm{H} 7$ cells $(50-60 \%$ confluent) were grown in $6-\mathrm{cm}$ culture dishes, and either $20 \mu 1$ of siRNA duplex $(20 \mu \mathrm{M})$ or a control duplex was introduced using Lipofectamine $2000^{\mathrm{TM}}$ (Invitrogen) according to the manufacturer's instruction and incubated for $24 \mathrm{~h}$ prior to experiments.

Quantitative real-time RT-PCR. Total RNA was isolated in Trizol $^{\mathrm{TM}}$ (Invitrogen), and template cDNA was synthesized from total RNA using the M-MLV reverse transcriptase $\mathrm{kit}^{\mathrm{TM}}$ (Invitrogen). Quantitative real-time PCR analysis was performed using the DNA engine Opticon2 system ${ }^{\mathrm{TM}}$ (BioRad Laboratories). cDNA product was amplified in a $20-\mu 1$ reaction containing a 10- $\mu 1$ DyNAmo $^{\mathrm{TM}}$ SYBR-Green qPCR kit (FINNZYMES, Espoo, Finland) and $1 \mu 1$ of each primer (5 mM). The primers for caveolin-1 were 5'-CGGGAACAG GGCAACATCTA-3' and 5'-TGTGTCCCTTCTTTCTGC-3', to amplify a 163-bp fragment corresponding to nucleotides 
A

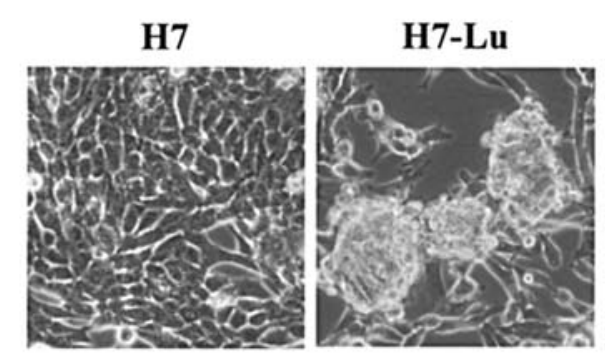

B

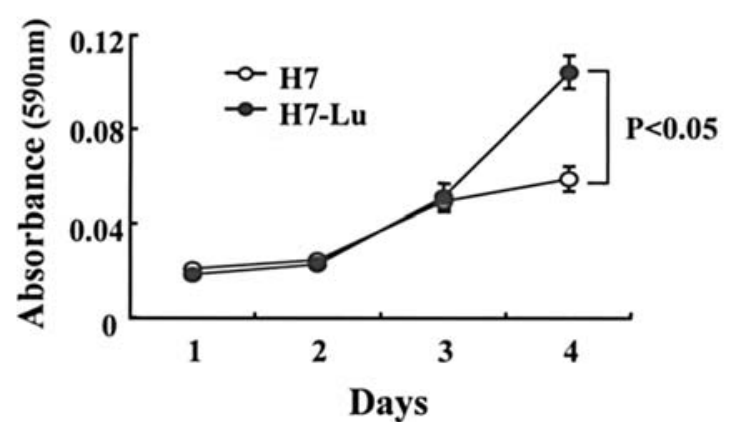

C

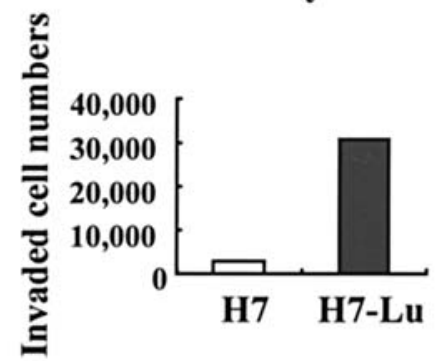

Figure 1. Establishment of a high-metastatic subline H7-Lu. H7, isolated from a low-metastatic subline of mouse Lewis lung cancer P29 was repeatedly injected into C57BL/6 mice with i.v. injection as described in Materials and methods. After five rounds of injection, a metastatic nodule in the lung was harvested to establish a high-metastatic subline $\mathrm{H} 7-\mathrm{Lu}$ in in vitro culture. (A) The morphology of H7 and H7-Lu cells was shown. (B) Results of the MTT assay to examine in vitro proliferation. The error bars represent SD $(n=3)$. P, Student's t-test. (C) Results of the invasion assay. Cells $\left(5 \times 10^{5}\right)$ were seeded into the upper chamber in the absence of serum and incubated for $20 \mathrm{~h}$, then the cells that appeared on the lower surface of the filter were fixed with $75 \%$ ethanol for $30 \mathrm{~min}$, then stained and counted under a microscope. Similar experiments were repeated three times with essentially the same results.

135-297 in the open reading frame (ORF) of the mouse caveolin-1 gene (GenBank). The PCR program consisted of initial denaturation at $95^{\circ} \mathrm{C}$ for 10 min followed by amplification for 40 cycles $\left(95^{\circ} \mathrm{C}\right.$ for $10 \mathrm{sec}, 60^{\circ} \mathrm{C}$ for $20 \mathrm{sec}$, and $72^{\circ} \mathrm{C}$ for $20 \mathrm{sec}$ ). Data were analyzed using Opticon Monitor2 software $^{\mathrm{TM}}$ (Bio-Rad Laboratories).

\section{Results}

Establishment of a high-metastatic subline. In order to establish high-metastatic sublines using a low-metastatic line of Lewis lung carcinoma (P29), we generated subline H7 from P29 by limiting dilution. Then, we repeatedly injected $\mathrm{H} 7$ into the tail veins of C57BL/6 mice. After five rounds of injection, a metastatic line H7-Lu was obtained. More than 200 metastatic foci were found on the lung surface in the case of $\mathrm{H} 7-\mathrm{Lu}$, while $\mathrm{H} 7$ parent cells showed only 2-5 foci. The morphology of these lines is shown in Fig. 1. H7-Lu cells were largely different from the parent $\mathrm{H} 7$ cells, i.e. frequently

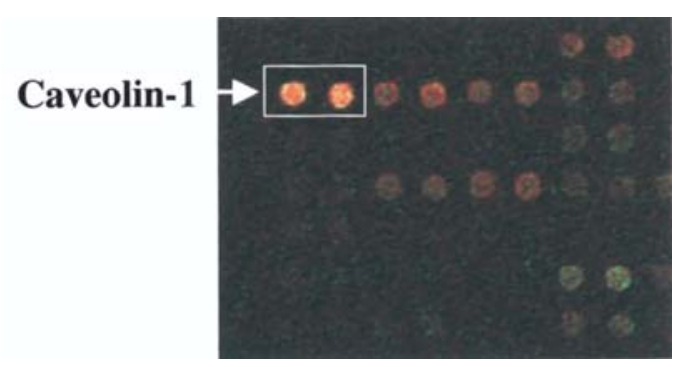

Figure 2. Gene expression profiling in $\mathrm{H} 7$ and H7-Lu cells. Signals of mouse caveolin-1 gene in the DNA microarray (white arrows). Cy3-dUTP (H7, red) or Cy5-dUTP (H7-Lu, green) was incorporated into cDNAs prepared from the mouse Lewis lung cancer cell sublines $\mathrm{H} 7$ and $\mathrm{H} 7-\mathrm{Lu}$, and hybridized with the DNA array. The spots corresponding to caveolin-1 are indicated in a part of the DNA array plate. The cDNA array had been prepared as duplicated spots.

A

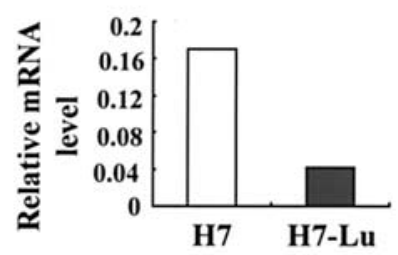

B

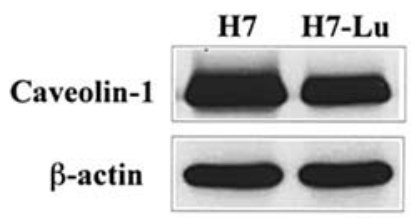

Figure 3. Reduced expression of mRNA and protein of caveolin-1 in H7-Lu. Expression levels of mRNA (A) and gene product (B) of caveolin-1 in H7 and H7-Lu cells were analyzed by quantitative real-time RT-PCR and immunoblotting, respectively.

aggregated and piled up. This subline showed higher proliferation and invasion activity than the parent line as shown in Fig. 1B and C, respectively. In particular, H7-Lu showed significantly higher cell growth than $\mathrm{H} 7$, suggesting that cells continued to proliferate even after reaching $100 \%$ confluency.

Gene expression profiling. Expression of genes in these two cell lines was analyzed using RNAs and DNA array containing more than 7,000 genes. Several genes showed marked downregulation. As shown in Fig. 2, the caveolin-1 gene was one of the markedly down-regulated genes in H7-Lu cells. Namely, the indeces were $0.26-0.35$ (fold). To confirm the reduced mRNA level of the caveolin-1 gene in H7-Lu, we examined caveolin-1 gene expression with real-time RT-PCR, showing a marked reduction in the mRNA level of the caveolin-1 gene in H7-Lu (Fig. 3A). With immunoblotting, definitely reduced caveolin-1 protein in H7-Lu was also demonstrated (Fig. 3B).

Effects of the knock-down of caveolin-1 on the properties of $H 7$ cells. Expression of the caveolin-1 gene was suppressed by knock-down with the siRNA technique. As shown in Fig. 4, 


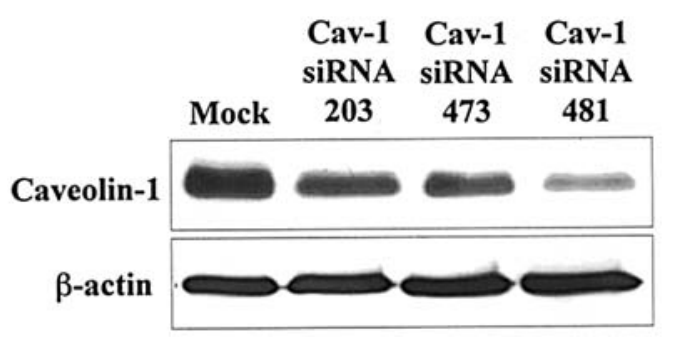

Figure 4. Knock-down of caveolin-1 in H7 cells. H7 cells were treated with nonspecific control fluorescein-labeled luciferase GL2 duplex (mock) or three siRNA duplex targeting sequences of mouse caveolin-1 nucleotides 203-228, 473-498, or 481-506 (Cav-1-siRNA). The expression of caveolin-1 was analyzed by immunoblotting. More than $80 \%$ knock-down of caveolin- 1 was obtained with siRNA 481-506. Lower panel bands of $\beta$-actin indicate that equal amounts of lysates were applied.

three siRNAs corresponding to 203 to 228,473 to 498 or 481 to 506 were tried, showing a reduction of band intensities of caveolin-1. Among the three siRNAs, the gene product was reduced in the transfectant cells (Cav-1-siRNA) of siRNA 481-506 to approximately $20 \%$ of that in the mock transfectant cells. Using the transient transfectant cells with this siRNA, the effects of the knock-down of caveolin-1 on the cell features were examined. In MTT assay, Cav-1-siNRA cells showed significantly increased cell proliferation from day 2 to day 4 after the siRNA transfection (Fig. 5A). In scratching wound healing assay, the cell motility of $\mathrm{H} 7$ transfected with caveolin-1 siRNA was also higher than that of mock-transfectant cells (Fig. 5B). In contrast, invasion activity as analyzed with the Matrigel/Boyden chamber was almost equivalent between Cav-1-siRNA cells and mocktransfected $\mathrm{H} 7$ cells (Fig. 5C).

Changes in the cellular signals in Cav-1-siRNA cells. Changes in the representative intracellular signals relevant to cell proliferation were examined in Cav-1-siRNA transfectant cells. As shown in Fig. 6A, the phosphorylation levels of ERK1/2 were higher in the Cav-1-siRNA cells compared to the mock control, particularly at 5 min after FBS treatment (Fig. 6A, lower). Phosphorylated Akt bands were also generally stronger in Cav-1-siRNA cells than in the control cells (Fig. 6B).

\section{Discussion}

Membrane microdomains such as GEM/rafts have been considered to be involved in the regulation of various cellular signals for cell proliferation, differentiation, and apoptosis (13). As a GEM/rafts marker, caveolins, flotillin (14) or ganglioside GM1 have been used in many studies (15). These molecules appear to be GEM/rafts markers, and play some roles in signal regulation. For caveolin families, caveolin-1 is most frequently down-regulated in many oncogenically transformed cells and tumors (16), although there has been some disagreement (10). Thus, caveolin-1 has been considered to be a tumor suppressor molecule. In fact, the suppression of caveolin-1 expression enhanced tumor development in vivo (17), and targeted disruption of the caveolin-1 gene also resulted in enhanced tumorigenesis $(18,19)$. Transfection of
A

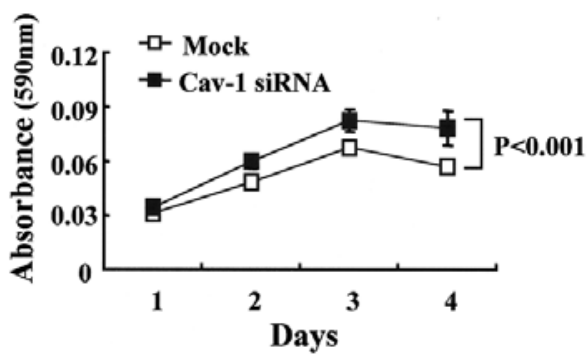

$\mathbf{B}$

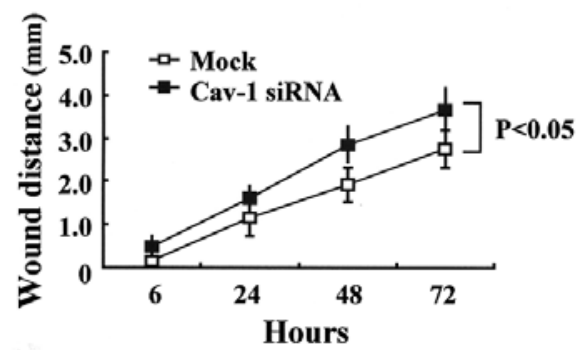

C

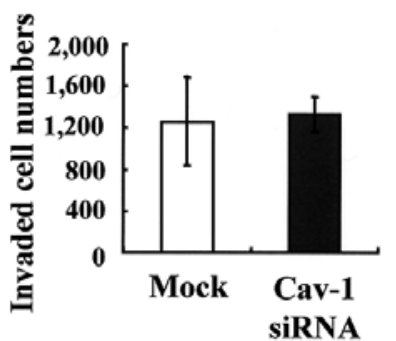

Figure 5. Effects of the knock-down of the caveolin-1 gene on the cell features. (A) The proliferation of Cav-1-siRNA cells in vitro was examined by MTT assay as described in Materials and methods. The error bars represent $\mathrm{SD}(\mathrm{n}=3)$. P, Student's t-test. (B) Results of the invasion assay showing the time course of the wound distances at the scratching-wounded spaces at 6, 24, 48 and $72 \mathrm{~h}$ after the scratching of the negative control (mock) or Cav-1-siRNA cells as indicated. The error bars represent SD $(n=6)$. P, Student's t-test. (C) Results of the invasion assay with the Boyden chamber. Cells $\left(5 \times 10^{5}\right)$ were seeded into the upper chamber in the presence or absence of serum and incubated for $20 \mathrm{~h}$ as described in Fig. 1C, then the cells that appeared on the lower surface of the filter were fixed with $75 \%$ ethanol, stained and counted under a microscope. The error bands represent $\operatorname{SD}(n=3)$.

GM1 synthase cDNA also resulted in the attenuation of cell proliferation in Swiss3T3 cells by modulating the GEM/rafts localization of the platelet-derived growth factor receptor (20). Overexpression of GM1 in NIH3T3 cells also induced changes in the intracellular local-ization of activated Ras proteins, resulting in the reduction of cell proliferation (21). Therefore, it seems reasonable that a high-metastatic subline H7-Lu showed downregulation of the caveolin-1 gene in the DNA array assay.

In the gene expression profiling, reduced expression levels of the caveolin-1 gene was one of the conspicuous changes in the high-metastatic P29 subline H7-Lu. Although a number of approaches to investigate the mechanisms for cancer metastasis have been performed using gene array techniques and many candidate genes involved in cancer metastasis have been identified $(22,23)$, it is not so easy to clarify whether those candidate genes are actually involved in the malignant properties of cancer cells. Interestingly, reduced caveolin-1 expression had profound implications for tumor properties such as cell proliferation and motility (Fig. 5). Thus, it was demonstrated that caveolin-1 is capable of modulating mole- 
A

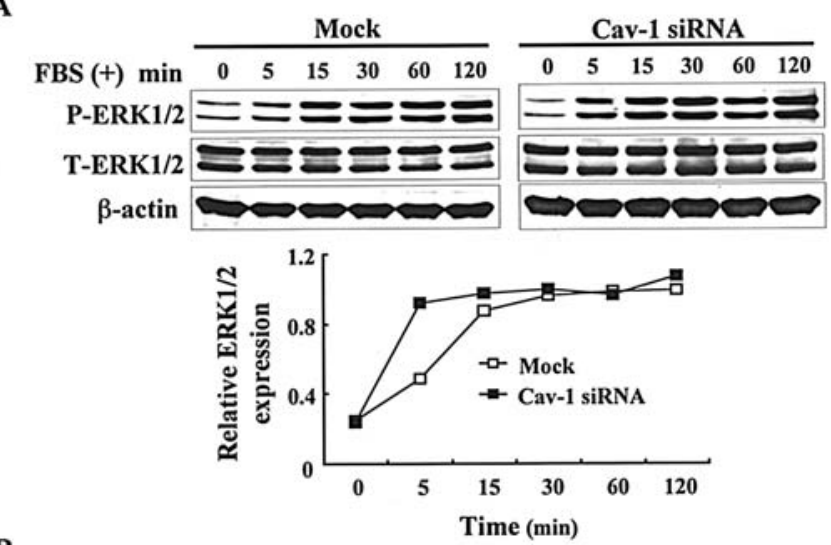

B

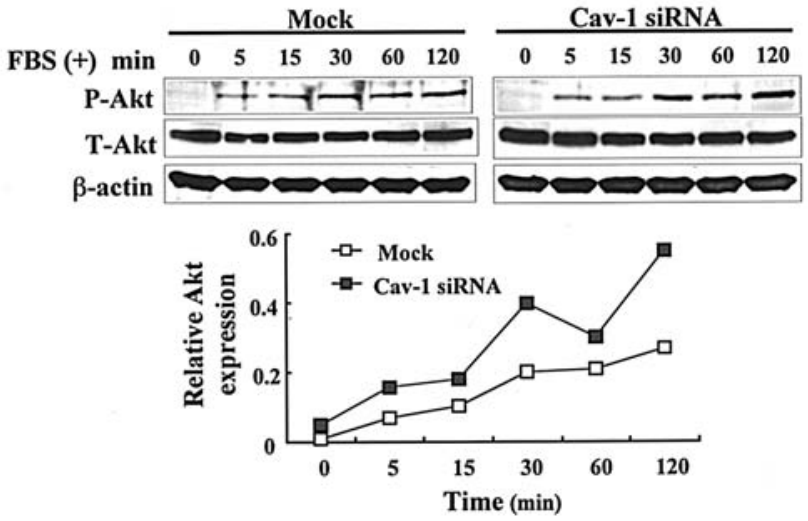

Figure 6. Effects of knock-down of caveolin-1 on the representative signals. (A) Results of immunoblotting for ERK1/2. Cells $\left(1 \times 10^{6}\right)$ were plated in 6-cm culture dishes and cultured in D-MEM containing 7.5\% FBS up to 70\% confluency followed by serum-starvation for $12 \mathrm{~h}$. Caveolin-1 siRNA (right) and negative control (mock) (left) cells were treated with FBS for the times indicated and then used for immunoblotting with an anti-phosphorylated ERK1/2 antibody (top). The intensity of bands was measured and corrected with those of total ERK1/2 (middle), and plotted (bottom). (B) Results of immunoblotting for the phosphorylation of Akt. Cells were treated as described in A, and then activation levels of Akt were analyzed by immunoblotting with an anti-phosphorylated Akt antibody (top), and band intensities were plotted after correction (bottom) with those of total Akt (middle).

cules directly or indirectly involved in tumor properties. Although H7-Lu showed increased invasion activity corresponding with its high incidence of lung metastasis, Cav-1siRNA cells showed no definite changes in the invasion activity in contrast with the results of the MTT assay and motility assay. These results indicate that the lowered expression level of caveolin-1 is involved in the increased high metastasis in vivo, and there should be other factors responsible for the increased invasion activity observed in H7-Lu. Other genes identified in our gene profiling analysis might contain such molecules.

The mechanisms with which caveolin-1 suppresses tumor properties in many cancer cells are not known. Therefore, identification of the molecules interacting with caveolin-1 in GEM/rafts should be critical to understand the way with which caveolin-1 exerts anti-tumor actions. We demonstrated both ERK1/2 and Akt activation levels increased in Cav-1-siRNA cells (Fig. 6), suggesting that signals transduced by growth factors/growth factor receptors were generally controlled by caveolin-1, and these signals were enhanced based on the modulation of GEM/rafts. Therefore, identification of the responsible factors present in FBS and its receptors expressed on the cell surface is quite important. In addition, the involvement of adhesion molecules such as integrins (24) and matrix metalloproteases (25) in tumor metastasis is also an important issue. Gangliosides GD2 and GD3 were reported to be involved in cell adhesion mediated by integrins (25) and the modulation of integrin functions with gangliosides was also reported by Wang et al (26) and by us (27). These issues remain to be investigated, particularly in the context of the modulation of GEM/rafts.

\section{Acknowledgements}

We thank Ms. Mizuno and Ms. Nakayasu for their technical assistance. This study was supported by a Grant-in-Aid for Scientific Research on Priority Area (14082102), and a Grantin-Aid for Scientific Research (16390075) from the Ministry of Education, Culture, Science, Sports and Technology of Japan, and by CREST, Japan Science and Technology Agency (JST).

\section{References}

1. Harlozinska A: Progress in molecular mechanisms of tumor metastasis and angiogenesis. Anticancer Res 25: 3327-3333, 2005.

2. Kannagi R: Molecular mechanism for cancer-associated induction of sialyl Lewis X and sialyl Lewis A expression - The Warburg effect revisited. Glycoconj J 20: 353-364, 2004.

3. Ellis LM: Angiogenesis and its role in colorectal tumor and metastasis formation. Semin Oncol 31: 3-9, 2004

4. Cao Y: Opinion: emerging mechanisms of tumour lymphangiogenesis and lymphatic metastasis. Nat Rev Cancer 5: 735-743, 2005.

5. Bjorklund M and Koivunen E: Gelatinase-mediated migration and invasion of cancer cells. Biochim Biophys Acta 1755: 37-69, 2005.

6. Brown DA and London E: Functions of lipid rafts in biological membranes. Annu Rev Cell Dev Biol 14: 111-136, 1998.

7. Stan RV: Structure of caveolae. Biochim Biophys Acta 1746: 334-348, 2005.

8. Simons K and Toomre D: Lipid rafts and signal transduction. Nat Rev Mol Cell Biol 1: 31-39, 2000.

9. Hakomori SI: Cell adhesion/recognition and signal transduction through glycosphingolipid microdomain. Glycoconj J 17: 143-151, 2000.

10. Williams TM and Lisanti MP: Caveolin-1 in oncogenic transformation, cancer, and metastasis. Am J Physiol Cell Physiol 288: 494-506, 2005

11. Nakanishi H, Oguri K Yoshida K, Itano N, Takenaga K, Kazama T, Yoshida A and Okayama M: Structural differences between heparan sulphates of proteoglycan involved in the formation of basement membranes in vivo by Lewis-lungcarcinoma-derived cloned cells with different metastatic potentials. Biochem J 288: 215-224, 1992.

12. Koike T, Kimura N, Miyazaki K, Yabuta T, Kumamoto K, Takenoshita S, Chen J, Kobayashi M, Hosokawa M, Taniguchi A, Kojima T, Ishida N, Kawakita M, Yamamoto H, Takematsu H, Suzuki A, Kozutsumi Y and Kannagi R: Hypoxia induces adhesion molecules on cancer cells: A missing link between Warburg effect and induction of selectin-ligand carbohydrates. Proc Natl Acad Sci USA 101: 8132-8137, 2004.

13. Hakomori S: Glycosylation defining cancer malignancy: new wine in an old bottle. Proc Natl Acad Sci USA 99: 10231-10233, 2002.

14. Parkin ET, Turner AJ and Hooper NM: Amyloid precursor protein, although partially detergent-insoluble in mouse cerebral cortex, behaves as an atypical lipid raft protein. Biochem J 344: 23-30, 1999.

15. Harder T, Scheiffele P, Verkade P and Simons K: Lipid domain structure of the plasma membrane revealed by patching of membrane components. J Cell Biol 141: 929-942, 1998. 
16. Koleske AJ, Baltimore D and Lisanti MP: Reduction of caveolin and caveolae in oncogenically transformed cells. Proc Natl Acad Sci USA 92: 1381-1385, 1995.

17. Galbiati F, Volonte D, Engelman JA, Watanabe G, Burk R, Pestell RG and Lisanti MP: Targeted downregulation of caveolin-1 is sufficient to drive cell transformation and hyperactivate the p42/44 MAP kinase cascade. EMBO J 17: 6633-6648, 1998

18. Razani B, Engelman JA, Wang XB, Schubert W, Zhang XL, Marks CB, Macaluso F, Russell RG, Li M, Pestell RG, Di Vizio D, Hou H Jr, Kneitz B, Lagaud G, Christ GJ, Edelmann W and Lisanti MP: Caveolin-1 null mice are viable but show evidence of hyperproliferative and vascular abnormalities. J Biol Chem 276: 38121-38138, 2001.

19. Williams TM, Lee H, Cheung MW, Cohen AW, Razani B, Iyengar P, Scherer PE, Pestell RG and Lisanti MP: Combined loss of INK4a and caveolin-1 synergistically enhances cell proliferation and oncogene-induced tumorigenesis: role of INK4a/CAV-1 in mammary epithelial cell hyperplasia. J Biol Chem 279: 24745-24756, 2004.

20. Mitsuda T, Furukawa K, Fukumoto S, Miyazaki H, Urano T and Furukawa K: Overexpression of ganglioside GM1 results in the dispersion of platelet-derived growth factor receptor from glycolipid-enriched microdomains and in the suppression of cell growth signals. J Biol Chem 277: 11239-11246, 2002.
21. Hamamura K, Tanahashi K, Furukawa K, Hattori T, Hattori H, Mizutani H, Ueda M, Urano T and Furukawa K: GM1 expression in H-ras-transformed NIH3T3 results in the suppression of cell proliferation inducing the partial transfer of activated H-ras from non-raft to raft fraction. Int J Oncol 26: 897-904, 2005.

22. Kozaki K, Koshikawa K, Tatematsu Y, Miyaishi O, Saito H, Hida T, Osada $\mathrm{H}$ and Takahashi T: Multi-faceted analyses of a highly metastatic human lung cancer cell line NCI-H460LNM35 suggest mimicry of inflammatory cells in metastasis. Oncogene 20: 4228-4234, 2001.

23. Bashyam MD: Understanding cancer metastasis: an urgent need for using differential gene expression analysis. Cancer 94: 1821-1829, 2002 .

24. Guo W and Giancotti FG: Integrin signalling during tumour progression. Nat Rev Mol Cell Biol 5: 816-826, 2004.

25. Cheresh DA, Pytela R, Pierschbacher MD, Klier FG, Ruoslahti E and Reisfeld RA: An Arg-Gly-Asp-directed receptor on the surface of human melanoma cells exists in divalent cationdependent functional complex with the disialoganglioside GD2. J Cell Biol 105: 1163-1173, 1987.

26. Wang XQ, Sun P and Paller AS: Ganglioside GM3 inhibits matrix metalloproteinase-9 activation and disrupts its association with integrin. J Biol Chem 278: 25591-25599, 2003.

27. Chen H-H, Fukumoto S, Furukawa K, Nakao A, Akiyama S, Urano T and Furukawa K: Suppression of lung metastasis of mouse Lewis lung cancer P29 with transfection of the ganglioside GM2/GD2 synthase gene. Int J Cancer 103: 169-176, 2003. 